\title{
Lesiones traumáticas en la mucosa oral de los adultos mayores
}

\section{Traumatic lesions of oral mucosa in older adults}

\author{
Somacarrera Pérez ML*, López Sánchez AF**, Martín Carreras-Presas C*, \\ Díaz Rodríguez $M^{*}$
}

\section{RESUMEN}

Dentro de la patología oral asociada con mayor frecuencia al paciente geriátrico nos encontramos con las lesiones con origen traumático. Los cambios fisiológicos que tienen lugar durante el envejecimiento hacen que las estructuras orales sean más susceptibles a la acción de agentes irritantes o agentes traumáticos, por lo que es importante establecer protocolos de prevención y diagnóstico precoz. En este artículo abordaremos las principales lesiones traumáticas que encontramos en el paciente anciano en función del tiempo de evolución, así clasificaremos las lesiones en agudas y crónicas.

Palabras clave: Mucosa oral, patología oral, lesiones traumáticas, paciente mayor, gerodontology.

\section{SUMMARY}

Among the oral pathology associated with geriatric patients we found more often we with traumatic injuries. The physiological changes that occurs during aging, makes that oral structures would be more susceptible to the action of irritants or traumatic agents, so it is important to establish different protocols for prevention and early diagnosis. In this paper we will review the major traumatic injuries found in the elderly patient depending on the time of evolution and so we classify the lesions in acute and chronic.

Key words: Oral mucosa, oral pathology, traumatic lesions, older patient, gerodontology.

Fecha de recepción: 2 de mayo 2015.

Aceptado para publicación: 6 de mayo 2015.

Somacarrera Pérez ML, López Sánchez AF, Martín Carreras-Presas C, Díaz Rodríguez M. Lesiones traumáticas en la mucosa oral de los adultos mayores. Av. Odontoestomatol 2015; 31 (3): 129-134.

* $\quad$ Facultad de Ciencias Biomédicas. Departamento de Odontología del Adulto. Universidad Europea de Madrid.

Carlos. Madrid.

\section{INTRODUCCIÓN}

Los traumatismos maxilofaciales suelen ser comunes en las primeras etapas de la vida, cuando aún no tenemos desarrolladas por completo nuestras habilidades motoras, y en la ancianidad, cuando éstas se ven mermadas $(1,2)$.

Los traumatismos en pacientes mayores de 65 años representan la $5^{\mathrm{a}}$ causa de mortalidad en la actuali- dad (1). Un tercio de las personas mayores de 75 años sufren alguna caída en casa anualmente, con fracturas u otros daños graves en la décima parte de estos accidentes.

Según un estudio realizado por Jainkittivong y cols. en el 2010, los pacientes ancianos presentaron lesiones asociadas a prótesis en el $45 \%$ de los casos, y aproximadamente el $60 \%$ de estos pacientes presentaba alguna lesión mucosa no asociada al uso de 
prótesis. Los pacientes portadores de dentaduras completas suelen presentar más lesiones que los pacientes portadores de dentaduras parciales removibles (3). En este tipo de pacientes es muy recomendable un tratamiento preventivo y una actuación precoz para que las consecuencias del traumatismo se conviertan en graves secuelas (2).

El paciente geriátrico es más susceptible a padecer lesiones más graves con traumatismos menos intensos que un paciente joven. A igualdad de intensidad de un traumatismo, un cuerpo joven regenera y recupera antes y mejor los tejidos dañados que un cuerpo anciano. Los pacientes gerontológicos presentan una cavidad oral con unas características que hacen más probables la aparición de lesiones con pérdida de sustancia que individuos de otros grupos etarios. Los cambios que pueden ocurrir con el envejecimiento en la mucosa oral se describen en la Tabla 1 (4). Todos estos cambios fisiológicos en muchos casos, y patológicos, secundarios a tratamientos de radioterapia o al uso de medicación xerostomizante, pueden hacer que el paciente anciano presente patología traumática con mayor probabilidad, convirtiendo su mucosa oral en susceptible a este tipo de lesiones.

En esta revisión, nos centraremos en las lesiones traumáticas que pueden afectar al paciente anciano. De forma aguda, cursando con pérdida de sustancia, o crónica, debida a la presencia de un agen-

\section{TABLA 1.- CAMBIOS EVOLUTIVOS EN LA}

CAVIDAD ORAL DEL PACIENTE ANCIANO. MODIFICADO DE BLANCO Y COLS. (4)

- Adelgazamiento y atrofia del epitelio.

- Aplanamiento interfase epitelio-tejido conectivo.

- Disminución de la velocidad metabólica y recambio celular.

- Disminución de las papilas filiformes.

- Desarrollo de lesiones vasculares y melánicas.

- Aumento de gránulos de Fordyce.

- Sialoadenosis y atrofia de glándulas salivales menores: Xerostomía. te irritante de manera continuada a lo largo del tiempo.

\section{LESIONES TRAUMÁTICAS AGUDAS}

La agresión producida en la mucosa oral por dientes, prótesis mal adaptadas, antiguas o rotas, y hábitos de introducir elementos extraños a la cavidad oral como palillos, bolígrafos puede desembocar en la aparición de distintas lesiones. De la misma manera, se pueden producir lesiones traumáticas por un cepillado agresivo $(5,6)$. Las clasificamos en erosiones y úlceras.

\section{a. Erosión aguda origen traumático}

Una erosión se define como una lesión por pérdida de sustancia superficial. Suele deberse a pequeños roces o traumatismos y no suele ser muy dolorosa, cicatrizando en 2 o 3 días sin dejar cicatriz, si el agente causal no se encuentra presente. Son lesiones en las que el agente causal suele ser fácilmente identificable y, por lo tanto, se puede intentar eliminar. Cuando existe fricción sobre la mucosa se podrá desprender de forma superficial el epitelio dando lugar a lesiones de tipo erosivo como la que se puede apreciar en la Figura 1.

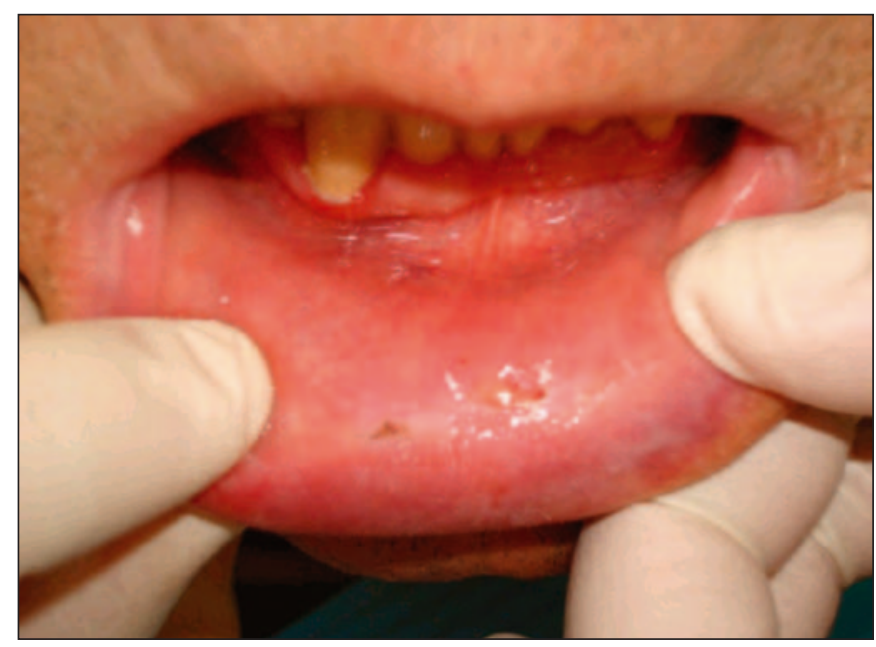

Fig. 1. Lesiones friccionales erosivas en un paciente anciano. Obsérvese como existe un patrón alternante entre lesiones con pérdida de sustancia y lesiones queratósicas, sugiriendo que agente causal se encuentra presente en la cavidad oral de manera crónica. 


\section{b. Úlcera aguda de origen traumático}

La úlcera traumática es una lesión frecuente en la mucosa oral. Consiste en una pérdida de sustancia en la que la lesión afecta a toda el espesor del epitelio. Estas lesiones, por lo general, suelen ser bastante dolorosas y pueden dejar cicatriz dependiendo normalmente de la extensión de las lesiones sobre todo en profundidad. Son lesiones reactivas en las que existe una relación causa-efecto identificable. Las úlceras traumáticas más comunes suelen producirse en zonas de oclusión. Deben eliminarse retenedores rotos, o reemplazar obturaciones antiguas que estén fracturadas o filtradas y tengan bordes cortantes que puedan irritar la mucosa. También interrogar acerca de hábitos alimentarios y parafuncionales (Fig. 2). Recordemos que la mucosa del paciente anciano suele ser más atrófica que en la juventud, de la misma manera que pueden presentar xerostomía, con la consecuente ausencia de lubricación y protección de la mucosa. Ejemplos de este tipo de lesiones son las mordeduras accidentales, con frecuencia producidas por caídas en el paciente mayor (Fig. 3).

Desde el punto de vista histopatológico son ulceraciones inespecíficas, con pérdida de continuidad del epitelio, un exudado fibrinoso que cubre el conectivo

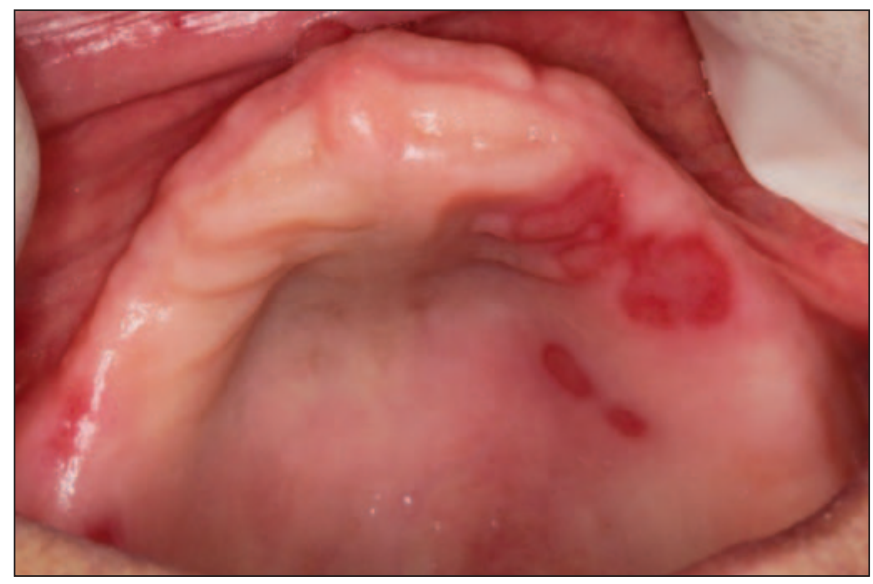

Fig. 2. Mujer de 80 años que acude por dolor en el paladar. A la exploración intraoral observamos que la paciente es desdentada total,

y no tiene prótesis para sustituir las piezas perdidas. Se aprecian múltiples úlceras, de bordes irregulares y halo eritematoso, distribuidas de manera no uniforme por el paladar duro. La paciente reconoció comer con la encía alimentos duros, tales como pan y patatas chips. Se aconsejó dieta blanda y aplicaciones de clorhexidina durante una semana. Las lesiones desaparecieron sin dejar cicatriz. expuesto un infiltrado inflamatorio a base de polimorfonucleares en las formas úlceras agudas y de linfocitos y células plasmáticas en las formas crónicas. Es fácil identificar en estas lesiones el origen que las ha producido. Si en dos semanas no curan al eliminar el posible agente etiológico, deben ser biopsiadas (7).

Las lesiones agudas por prótesis suelen deberse a la presencia de aletas sobreextendidas. En este caso, la lesión desaparecerá al rebajar el contorno de la zona traumatizada. De las lesiones no asociadas a prótesis, las más prevalentes son las úlceras traumáticas. Martori y cols. (8) publican en su estudio que, de las lesiones asociadas a prótesis en una población de ancianos estudiada, el $34 \%$ eran queilitis angulares, el $15 \%$ eran alguna úlcera asociada a la prótesis y un $14 \%$ presentaban candidiasis protésica. Además, según este estudio, el $54 \%$ de los pacientes estudiados presentaban al menos una lesión relacionada con la prótesis removible. Las úlceras fueron más comunes en pacientes con grandes atrofias maxilares y reabsorciones alveolares (8) (Fig.4).

Así mismo debe valorarse el ajuste correcto y buena adaptación de la prótesis que no permita un balanceo excesivo, y debe ajustarse la oclusión.

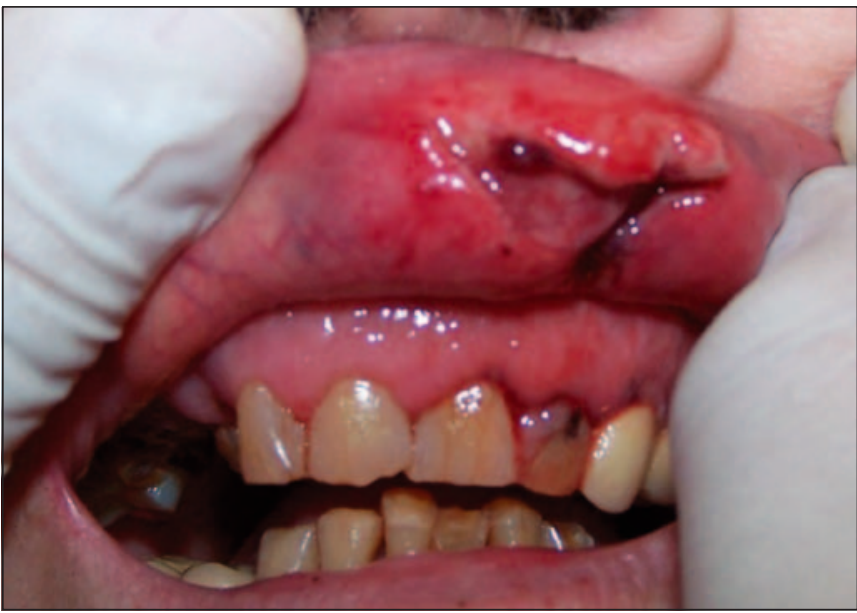

Fig. 3. Paciente de 75 años, institucionalizada en un centro para mayores por padecer Alzheimer en estado avanzado. El personal auxiliar que le acompaña a la clínica refiere que la paciente se había caído de la cama. A la exploración extraoral, se observaron múltiples equimosis a nivel labial y facial. En la exploración intraoral,

observamos una úlcera de bordes irregulares y amplia respuesta inflamatoria. Así mismo se pueden observar lesiones a nivel dentario. 


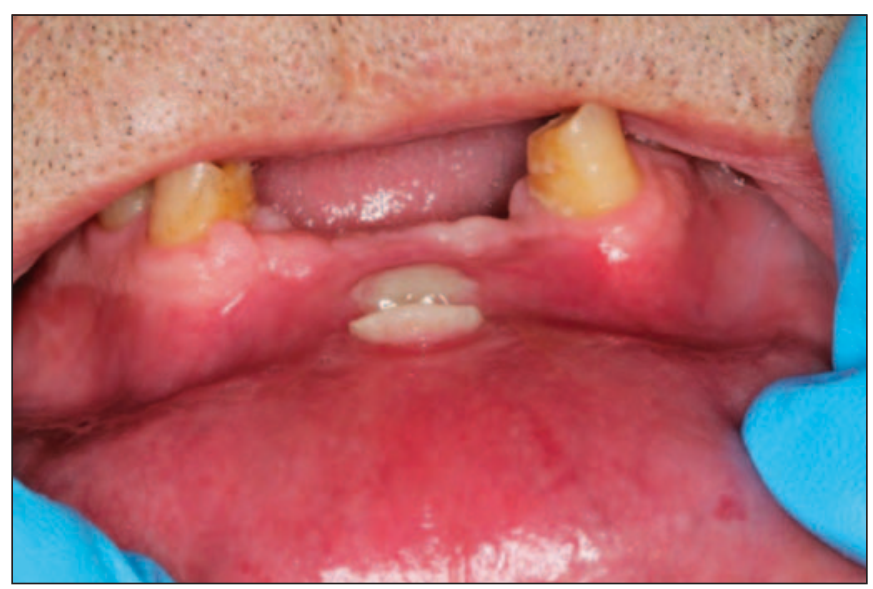

Fig. 4. Paciente varón de 69 años que presenta una úlcera asociada a una aleta vestibular de una prótesis removible. Se ajustaron los retenedores y se alivió la zona del acrílico. Se prescribió clorhexidina en gel al 0,2\%. La lesión cicatrizó en una semana sin dejar cicatriz.

\section{LESIONES TRAUMÁTICAS CRÓNICAS}

Cuando el agente irritante persiste en el tiempo, se producen lesiones crónicas que se pueden clasificar en: hiperqueratosis friccional, morsicatio buccarum, fibroma, hiperplasia papilar inflamatoria y epulis fissuratum.

\section{a. Hiperqueratosis friccional}

La fricción o acción mecánica de roce continuado de baja intensidad sobre la mucosa oral produce una reacción de defensa del epitelio, que resulta en un aumento de la capa de queratina, dando lugar a lesiones de color blanco (queratosis friccionales). Esta respuesta protectora de la mucosa a nivel microscópico, genera una lesión blanca en el aspecto macroscópico. Las lesiones blancas friccionales, no se desprenden al raspado, son asintomáticas y en ellas se puede demostrar una clara relación causa-efecto (9). Se encuentran frecuentemente en zonas de roce, en espacios o brechas edéntulas o en zonas de oclusión dentaria (Fig. 5). Su diagnóstico es clínico y en el diagnóstico diferencial deberemos incluir otras lesiones de aspecto blanquecino como el liquen, buscando lesiones en otras localizaciones de forma bilateral, o la leucoplasia. En la mucosa yugal también puede observarse la presencia de la línea alba, que consiste en la huella de los dientes sobre la mucosa

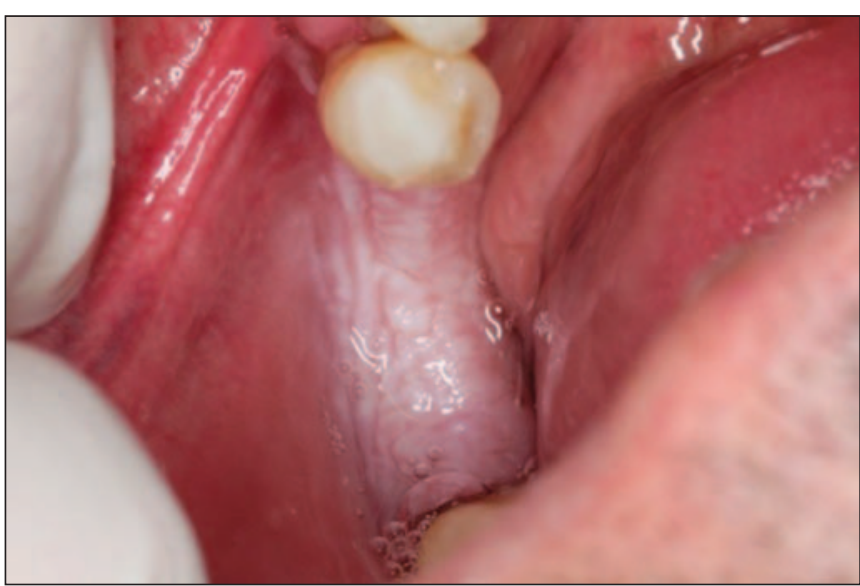

Fig. 5. Placa blanca, que no se desprende al raspado, de aspecto fibroso, en una brecha edéntula del tercer cuadrante de un paciente geriátrico. Las lesiones son asintomáticas.

de la mejilla, en el plano de oclusión, no considerándose una patología propiamente dicha, más que una variación no patológica de la normalidad.

\section{b. Morsicatio buccarum}

El morsicatio buccarum consiste en lesiones blanquecinas esfaceladas por mordisqueo de la mucosa yugal o labial (Fig. 6). Se trataría de un hábito parafuncional, en el que un paciente generalmente ansioso, se mordisquea la mucosa de manera compulsiva (10).

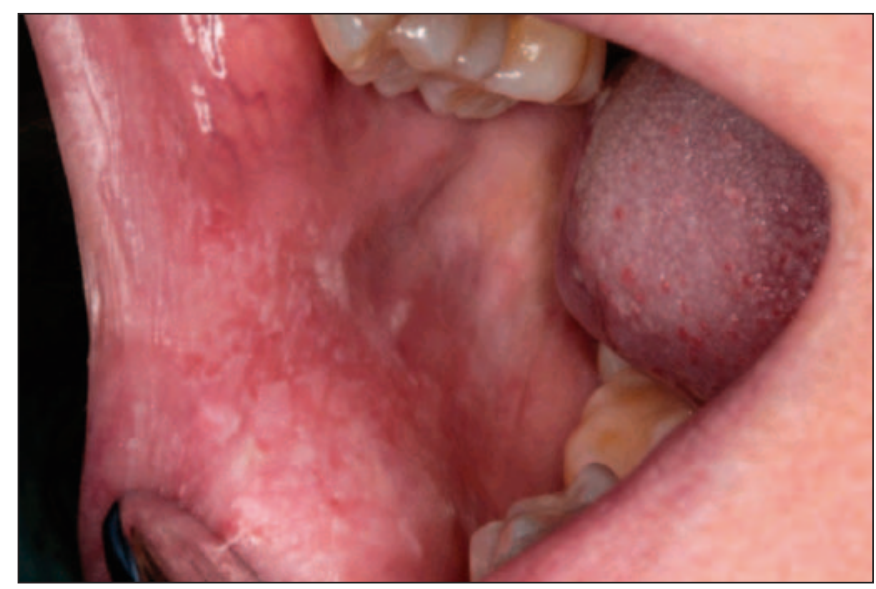

Fig. 6. Morsicatio buccarum en una paciente de 68 años. Obsérvese el patrón alternante típico de erosiones y zonas hiperqueratósicas. El aspecto de las lesiones es rugoso y heterogéneo. Suelen ser asintomáticos. 
El mecanismo de acción de estas lesiones es por fricción de los dientes sobre la mucosa de manera continuada y compulsiva, asociada a un estado de ansiedad.

\section{c. Fibroma traumático}

Los fibromas son lesiones exofíticas, bien delimitadas, localizadas en una zona de fricción. Generalmente, no presentan sintomatología asociada, pero en ocasiones, si existe una proliferación nerviosa sensitiva reactiva las lesiones podrían ser dolorosas, denominándose neurofibromas. Suelen presentar el mismo color que la mucosa oral subyacente y tienen consistencia fibrosa y firme. Son lesiones reactivas ante un traumatismo (Fig. 7). Las lesiones producidas por agentes mecánicos son fáciles de diagnosticar tras una adecuada historia clínica y exploración, indagando en la relación entre el factor causal y el efecto provocado. Eliminar el factor causal será el tratamiento idóneo $(4,8,11)$.

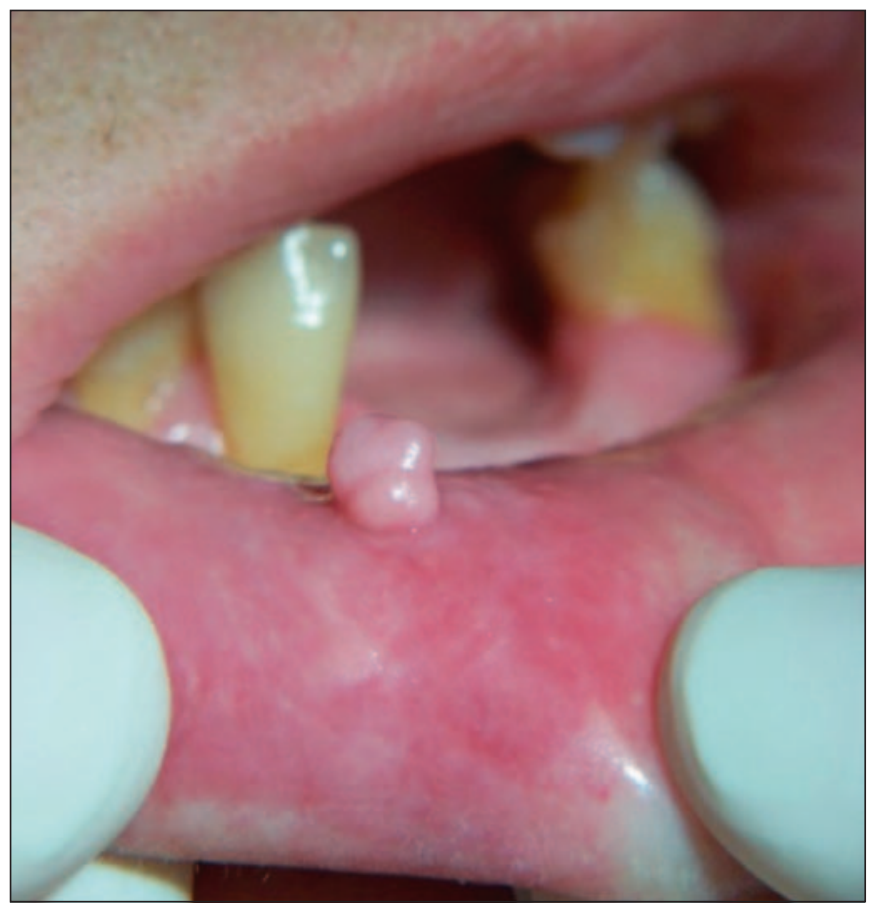

Fig. 7. Lesión exofítica de aspecto benigno localizada en la mucosa labial interna, de varios años de evolución. La lesión es dura a la palpación, y circunscrita. La paciente refiere morderse esa zona con frecuencia. Su tratamiento consiste en la extirpación quirúrgica de la lesión, en este caso mediante biopsia excisional.

\section{d. Hiperplasia papilar inflamatoria}

Se suele presentar en pacientes portadores de prótesis, aunque se han descrito casos en respiradores orales. Se trata de un aumento de volumen en forma de proyecciones papilares. Su consistencia es blanda, salvo si el tiempo de evolución es extenso, que las lesiones tienden a fibrosarse. En ocasiones se ulceran y pueden sangrar. Antiguamente, se confeccionaban las prótesis con un reservorio en la bóveda palatina (succionador), en forma de corazón, que se pensaba facilitaba la retención de la prótesis por succión. Hoy en día, sabemos que este tipo de diseños puede producir lesiones en la fibromucosa palatina, también hiperplásicas, en las que el negativo de la prótesis se puede observar en la mucosa palatina (12).

\section{e. Epulis fissuratum}

Son lesiones crónicas reactivas a un mal ajuste de prótesis. Generalmente se trata de prótesis antiguas, mal adaptadas, con procesos alveolares reabsorbidos, pero también se pueden observar en prótesis nuevas con aletas o faldones excesivamente largos (Fig. 8). En general, suelen ser asintomáticas y de coloración similar a la mucosa subyacente. Suelen ser lesiones fibrosas que siguen el contorno de una aleta o una prótesis desadaptada. El tratamiento de

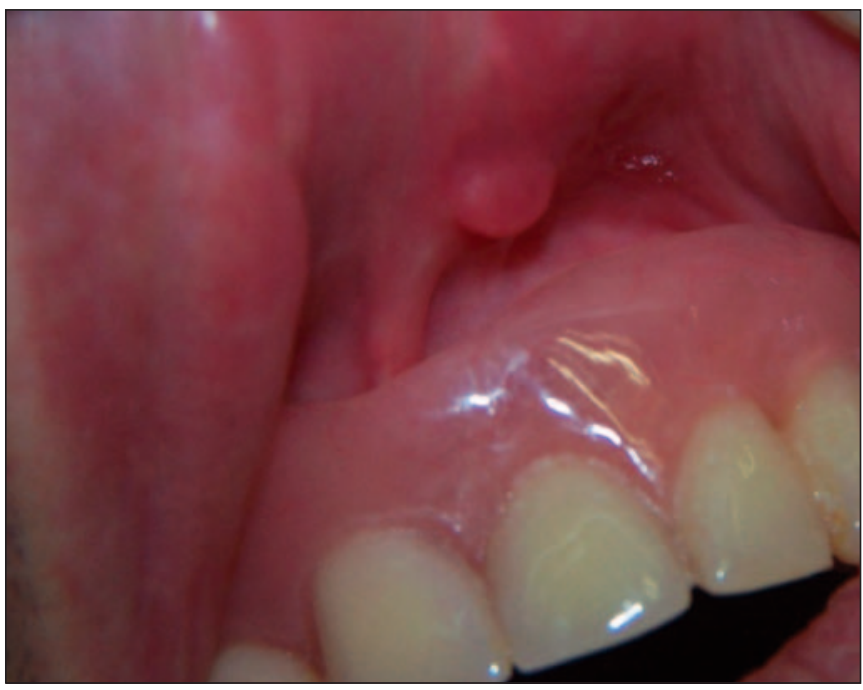

Fig. 8. Lesión exofítica crónica, fibrosa a la palpación, asociada a la aleta vestibular de una prótesis mal adaptada. 
este tipo de lesiones consiste en eliminar el tejido alterado y realizar un correcto rebase y reajuste de la prótesis si fuera posible o confeccionar una prótesis nueva en caso de desajustes muy grandes o prótesis muy antiguas $(12,13)$.

\section{BIBLIOGRAFÍA}

1. Varas-Fabra F, Castro Martín E, Pérula de Torres LA, Fernández Fernández MJ, Ruiz Moral R, Enciso Berge I. Falls in the elderly in the community: prevalence, consequences, and associated factors. Aten Primaria 2006;38:450-5.

2. Moreno-Martínez NR, Ruiz-Hidalgo D, BurdoyJoaquim E, Vásquez-Mata G. Incidencia y factores explicativos de las caídas en ancianos que viven en la comunidad. Rev Esp Geriatr Gerontol 2005;40:11-7.

3. Jainkittivong A, Aneksuk V, Langlais RP. Oral mucosal lesions in denture wearers. Gerodontology 2010;27:26-32.

4. Blanco A, Gándara JM, Gamallo A. Patología de la mucosa oral. En: Chimenos E, Ribera M, López J. Gerodontología. $1^{\text {a }}$ Ed. Santiago de Compostela: SEGER 2012;185-204.

5. Beauvillain de Montreuil C, Tessier MH, Billet P. Patologia benigna della mucosa orale. EMCOtorinolaringoiatria 2012;11:1-20.

6. Velasco E, Obando R, Vigo M, Martínez-Sahuquillo A, Bullón P. Valoración de la mucosa oral en pacientes geriátricos. Av Odontoestomatol 1995;9:691-700.
7. Díaz Y, de la Caridad I, Zamora J. Afecciones de la mucosa oral encontradas en pacientes geriátricos portadores de prótesis estomatológicas. Rev Cubana Estomatol 2007;44:3-7.

8. Martori, E, Ayuso-Montero, R, Martinez-Gomis, $J$, Viñas, M, Peraire, $M$. Risk factors for denturerelated oral mucosal lesions in a geriatric population J Prosthet Dent 2014;111:273-9.

9. Sook-Bin Woo. Atlas de Patología Oral. Rio de Janeiro: Elsevier 2013.

10. Bagán JV. Medicina y Patología Bucal. $1^{\text {a }}$ ed. Madrid: Axon 2013.

11. Scully C. Oral and Maxillofacial Medicine. $3^{a} \mathrm{Ed}$. Londres: Churchill Livingstone 2013.

12. Coelho CM, Zucoloto S, Lopes RA. Dentureinduced fibrous inflammatory hyperplasia: a retrospective study in a school of dentistry. Int $\mathrm{J}$ Prosthodont 2000;13:148-51.

13. Coelho C, Sousa Y, Daré A. Denture-related oral mucosal lesions in a Brazilian school of dentistry. J Oral Rehabil 2004;31:135-9.

\section{CORRESPONDENCIA}

Dra. María Luisa Somacarrera Pérez

Departamento de Odontología.

Facultad de Ciencias Biomédicas.

Universidad Europea de Madrid.

28670 Villaviciosa de Odón, Madrid

Correo electrónico: mluisa.somacarrera@uem.es 